\title{
Conventional cervical smears were better than monolayer cytology or human papillomavirus testing for detecting cervical intraepithelial neoplasia
}

Coste J, Cochand-Priollet B, de Cremoux P, et al. Cross sectional study of conventional cervical smear, monolayer cytology, and human papillomavirus DNA testing for cervical cancer screening. BMJ 2003;326:733-6.

\section{QUESTION: In women referred for colposcopy or presenting for routine smears, is monolayer cytology (MLC) or human papillomavirus (HPV) testing more accurate than the conventional cervical smear (CCS) test for detecting cervical neoplasia?}

\section{Design}

Blinded comparison of the CCS test, MLC, and HPV testing with colposcopy and histology as the reference standard.

\section{Setting}

2 university centres and 2 private practices in France.

\section{Participants}

828 women (mean age 38 y) who had been referred for colposcopy and 1757 women (mean age 33 y) who presented for routine smears.

\section{Description of tests and diagnostic standard}

All women were evaluated by the 3 methods (CCS test, MLC, and HPV testing), and by colposcopy followed by biopsy if abnormalities were detected. After the CCS test, the remaining portion of the sample was used for the monolayer slide and for HPV testing. Smear abnormalities were classified into 5 ordered categories (negative, atypical squamous/glandular cells of undetermined significance, low or high grade squamous intraepithelial lesions, and invasive cancer), and the reference standard was classified into 4 ordered categories (normal colposcopy or negative biopsy result; cervical intraepithelial neoplasia $[\mathrm{CIN}]$ grades I, II, and III; and invasive carcinoma). The clinical readings and optimised interpretations (2 blinded readings followed, if necessary, by consensus) were done by experienced cytopathologists.

\section{Main outcome measures}

Sensitivity and specificity, and positive and negative likelihood ratios.

\section{Main results}

$65 \%$ of women referred for colposcopy and $5.8 \%$ of women presenting for routine smears had cervical intraepithelial neoplasia ( $\geq$ CIN I). Sensitivity and specificity, and positive and negative likelihood ratios for the 3 methods are in the table.

\section{Conclusion}

In women referred for colposcopy or presenting for routine smears, the conventional cervical smear test was more accurate than monolayer cytology or human papillomavirus testing for detecting cervical neoplasia.

\section{COMMENTARY} nostic study that avoided verification bias. document this finding in different age groups. HPV testing in women with equivocal papanicolaou smears is growing. ${ }^{1}$ cancer precursors. Arch Pathol Lab Med 2003;127:950-8.
Sources of funding: Direction Générale de la Santé and

Programme Hospitalier de Recherche Clinique, French Ministry of Health and Association pour la Recherche sur le Cancer.

For correspondence: Professor J Coste,

Hopital Cochin, Paris, France.

coste@

cochin.univ-paris5.fr

Abstract and commentary also appear in ACP Journal Club

Coste et al compared cytological results from the CCS test, MLC, and high risk HPV testing with the gold standard (colposcopy and biopsy if indicated) in women referred for colposcopy or presenting for routine smears. Thus, they performed a standard diag-

It should be noted, however, that the cytologists were initially inexperienced in MLC and that the MLC smear was prepared from the sample that remained after producing the CCS test. Such a fixed sequence may introduce some bias at the expense of MLC.

The authors conclude that the CCS test consistently had better sensitivity and specificity than MLC for detecting CIN $\geq$ I or CIN $\geq$ II. However, an overlap of the confidence intervals weakens the strength of this claim. In our view, these results do not support any meaningful differences in diagnostic indicators between the 2 cytology systems. They support worse results for HPV testing at detecting CIN $\geq$ I (in women referred for colposcopy) but better results for detecting CIN $\geq \mathrm{II}$ in women who presented for screening (sensitivity for HPV testing was $96 \%$, whereas that for the CCS test at a threshold $\geq$ high grade squamous intraepithelial lesions was only $51 \%$; although HPV testing had considerably lower specificity). It might be interesting to

Although the study was rigorously done, its results will have to be considered together with findings from other studies. The evidence to support any firm choice between the CCS test and MLC is still insufficient. For HPV testing, the picture may be different. The initial expectations of good performance with respect to initial screening are not being confirmed. However, interest as well as the supporting evidence that favours triage with

Frank Buntinx, MD, PhD

University of Leuven

Leuven, Belgium and Maastricht, The Netherlands

Marc Arbyn, MD, MSc Scientific Institute of Public Health Brussels, Belgium

1 Cox JT, American Society for Colposcopy and Cervical Pathology. The clinician's view: role of human papillomavirus testing in the American Society for Colposcopy and Cervical Pathology Guidelines for the management of abnormal cervical cytology and cervical

Diagnostic properties of the conventional cervical smear (CCS) test, monolayer cytology (MLC), and human papillomavirus (HPV) testing for detecting cervical intraepithetial neoplasia*

\begin{tabular}{|c|c|c|c|c|c|c|c|c|}
\hline \multirow[t]{2}{*}{ Test } & \multicolumn{4}{|c|}{ Women referred for colposcopy } & \multicolumn{4}{|c|}{ Women presenting for routine smears } \\
\hline & $\begin{array}{l}\text { Sensitivity } \\
\text { (95\% CI) }\end{array}$ & Specificity (CI) & + LR & - LR & Sensitivity (CI) & Specificity (CI) & +LR & $-\mathbf{L R}$ \\
\hline MLC† & $92 \%(90$ to 95$)$ & $58 \%(52$ to 64$)$ & 2.2 & 0.1 & $66 \%(56$ to 75$)$ & $91 \%$ (90 to 93$)$ & 7.5 & 0.4 \\
\hline HPV testing & $82 \%(77$ to 86$)$ & $74 \%(67$ to 80$)$ & 3.1 & 0.3 & $69 \%$ (58 to 79$)$ & $83 \%(81$ to 85$)$ & 4.1 & 0.4 \\
\hline
\end{tabular}

${ }^{*}$ Abnormality threshold $=$ atypical squamous cells or glandular cells of undetermined significance for CCS test and MLC, and relative light units $>1$ for HPV testing. Diagnostic terms defined in glossary. †Clinical reading. 